\title{
La diffusion des cours universitaires en direct : retour sur une ancienne nouveauté ${ }^{1}$
}

\author{
Live streaming for university courses: a look back at an old \\ novelty
}

\section{Transmitir cursos universitarios en directo: una mirada retrospectiva a una antigua novedad}

Claire Peltier, professeure adjointe

Université Laval, Canada

claire.peltier@fse.ulaval.ca

RÉSUMÉ

La diffusion des cours en direct est une tendance qui se dessine depuis quelques années dans l'enseignement supérieur et a constitué une solution de choix pour la mise à distance des cours universitaires durant la pandémie de COVID-19. Présentée comme une innovation, la diffusion des cours en direct est pourtant loin d'être une nouveauté. Cet article se propose d'aborder l'usage pédagogique de la captation vidéo à des fins de transmission et de diffusion des savoirs sous l'angle de l'histoire de la formation à distance et des technologies éducatives, ainsi que sous celui de l'innovation pédagogique. L'exemple de la mise en place, à travers trois cours pilotes, d'un dispositif de diffusion des cours en direct à l'Université de Genève sera analysé à l'aune des changements potentiels engendrés par l'introduction d'une nouveauté dans la routine des pratiques.

Mots-clés : diffusion des cours en direct, formation à distance, technologies éducatives, usage pédagogique de la vidéo, innovation pédagogique, enseignement supérieur 
Live streaming has been a trend in higher education for several years and was a preferred solution for the distance delivery of university courses during the COVID-19 pandemic. Although presented as an innovation, live streaming is far from being a novelty. This article proposes to approach the pedagogical use of video to transmit and disseminate knowledge from the perspective of the history of distance learning and educational technologies and that of pedagogical innovation. The example of implementing a live lecture broadcasting system at the University of Geneva will be analyzed in light of the potential changes brought about by introducing a novelty in the routine of practices.

Keywords: live streaming, distance learning, educational technologies, pedagogical use of video, pedagogical innovation, higher education

RESUMEN

La retransmisión en directo ha sido una tendencia en la enseñanza superior durante los últimos años y fue la solución preferida para impartir cursos universitarios a distancia durante la pandemia de COVID-19. Aunque se presenta como una innovación, la transmisión en directo está lejos de ser una novedad. Este artículo propone abordar el uso pedagógico del vídeo para la transmisión y la difusión de conocimientos desde la perspectiva de la historia de la formación a distancia y de las tecnologías educativas, así como de la innovación pedagógica. El ejemplo de implantación de un sistema de transmisión en directo de cursos en la Universidad de Ginebra se analizará considerando los posibles cambios generados por la introducción de una novedad en la rutina de las prácticas.

Palabras clave: difusión de cursos en directo, formación a distancia, tecnologías educativas, uso pedagógico del vídeo, innovación pedagógica, educación superior

\section{Introduction}

Le monde des technologies éducatives est "pauvre en mémoire ", disait Alain Chaptal (1996). Cette formule illustre l'éternel recommencement auquel est soumise la sphère éducative dès lors qu'il s'agit de questionner l'usage des technologies numériques. Ce "phénomène cyclique d'enchantementdésenchantement ${ }^{2}$, caractéristique de l'usage des technologies éducatives, se traduit par différentes phases : tout d'abord un enthousiasme initial attribuant à la nouveauté technologique de nombreuses vertus, puis une certaine forme de déception due à des attentes non remplies ou à des difficultés inattendues, enfin la critique, voire le rejet, d'une innovation qui n'a pas tenu ses promesses. La méconnaissance de l'histoire des technologies éducatives et, plus largement, de la formation à distance,

${ }^{2}$ Chaptal fait ici référence aux travaux de Larry Cuban (1986, notamment). 
associée à une certaine forme de technocentrisme ${ }^{3}$ permet d'expliquer bon nombre d'« échecs » en la matière.

L'engouement actuel autour de l'usage de la vidéo dans l'éducation, notamment dans l'enseignement supérieur $(M O O C$, classes inversées par exemple), n'échappe pas à cette règle. Pour illustrer cette dynamique, nous prendrons appui sur une autre tendance, en lien avec l'usage de la vidéo, qui émerge depuis les années 2000 : celle de la diffusion de cours en direct (live streaming en anglais) (Woolfitt, 2015; O'Callaghan, Neumann, Jones et Creed, 2017). Depuis 2016, I'Université de Genève (Suisse) a progressivement ${ }^{4}$ mis en place un tel dispositif pour certains enseignements de première année de baccalauréat universitaire afin de répondre à une problématique récurrente d'augmentation des effectifs étudiants et de manque de place dans les salles de cours. La diffusion de cours en direct était initialement présentée comme une innovation ${ }^{5}$. Toutefois, la nature de cette innovation demande à être questionnée. À quel type d'innovation avons-nous affaire ? S'agit-il uniquement d'une innovation technologique destinée à répondre à une difficulté d'ordre logistique et organisationnelle? Peut-il s'agir d'une innovation (techno)pédagogique? À quelles conditions pourrait-on la considérer comme telle? C'est à ces différentes questions que nous tenterons de répondre, tout en faisant référence à la continuité sociohistorique dans laquelle s'inscrit l'usage de la vidéo à des fins pédagogiques. Afin de situer la problématique à laquelle répond la mise en place d'un dispositif tel que la diffusion des cours en direct, nous aborderons tout d'abord la question de la démocratisation et de la massification dans l'enseignement supérieur. Nous poursuivrons avec quelques éléments de réflexion autour de l'innovation pédagogique avant d'évoquer l'histoire de la télédiffusion des cours universitaires. L'exemple de la mise en place du service de live streaming à I'Université de Genève sera ensuite analysé à l'aune de la question de l'innovation pédagogique initialement évoquée, avant de conclure sur la nécessité de redonner une mémoire aux domaines de la formation à distance et des technologies éducatives, à l'heure où celles-ci sont sous le feu de nombreuses critiques.

\section{Démocratisation et massification de l'enseignement supérieur}

La massification de la population universitaire constitue un phénomène généralisé dans la plupart des pays occidentaux depuis les années 1980 (Charle et Verger, 2012). Les réformes menées en Europe pendant les années 1990-2000, notamment dans le cadre du processus de Bologne, ont contribué à l'internationalisation des études, à la mobilité des étudiants, ainsi qu'à l'accroissement des effectifs. L'augmentation de la population étudiante est susceptible de poser un certain nombre de problèmes logistiques, notamment en matière de confort et de sécurité. Le nombre de places disponibles dans les salles de cours est l'un d'entre eux. Cette difficulté récurrente oblige les établissements d'enseignement supérieur à trouver des solutions. La massification ne s'appréhende toutefois pas uniquement à l'aune de l'augmentation de la population étudiante. Elle se traduit aussi par une grande hétérogénéité à la fois

\footnotetext{
${ }^{3}$ Par « technocentrisme ", on peut entendre la propension à attribuer à la technologie toutes les vertus et à minimiser l'influence des facteurs humains dans les effets escomptés et constatés (pour plus de détails sur l'opposition technocentrisme/anthropocentrisme dans l'activité médiatisée, voir Rabardel, 1995)

${ }^{4}$ La mise à distance de l'ensemble des cours durant le printemps et l'automne 2020, imposée par la crise sanitaire, a précipité sa généralisation.

${ }^{5}$ https://www.unige.ch/lejournal/numeros/journal160/article-point-fort/
}

revue-mediations.teluq.ca | № 6, 2021 
sociale, culturelle et économique (Gruel, Galland et Houzel (dir.), 2009, cités par Endrizzi et Sibut, 2015, p. 3). Dans ce contexte, repenser l'enseignement pour l'ajuster à cette nouvelle réalité s'impose comme une nécessité. Pourtant, comme le souligne Albero (2014, p. 28) :

Quand [la pédagogie universitaire] s'institutionnalise, c'est dans sa seule perspective pratique, en vue de résoudre des problèmes de gestion des activités de flux d'étudiants et développer des ressources appropriées. Les solutions attendues sont principalement fonctionnelles, locales, économiques, peu perturbantes pour les structures et les habitudes.

C'est ainsi que sont souvent confondues innovations technologiques et innovations pédagogiques. Loin d'être mutuellement exclusives, ces deux formes d'innovation et les conditions de leur articulation méritent d'être regardées de plus près.

\section{L'innovation technopédagogique dans l'enseignement supérieur}

La question générale de l'innovation et celle, plus spécifique, de l'innovation pédagogique ont fait l'objet de longue date d'une nombreuse littérature dans différents domaines disciplinaires (économie, ingénierie, sociologie, éducation, etc. $)^{6}$. La problématique de l'articulation de la technologie, de la pédagogie et de l'innovation dans l'enseignement supérieur a également donné lieu à une production scientifique importante ${ }^{7}$.

Dans sa recension des écrits relatifs à l'enseignement supérieur et aux innovations pédagogiques, Béchard (2001, p. 258) évoque la distinction opérée par Cros et Adamczewski (1996) entre réforme (spécifiquement liée à des critères « d'efficacité et de rentabilité »), novation (" liée à l'objet, à l'œuvre ou au produit ») et innovation, laquelle "s'inscrit davantage dans un processus campé dans un contexte donné ». Pour aller plus loin dans cette définition, le critère d'amélioration substantielle des apprentissages des étudiants amené par Bédard et Béchard (2009) paraît pertinent à prendre en compte. Ce critère pose toutefois la question de son évaluation et des indicateurs de référence pour mesurer cette amélioration. II s'agit à cet égard de dépasser les traditionnelles études comparatives " avec et sans " menées généralement en mode expérimental ou quasi expérimental et qui, le plus souvent, amènent le chercheur à la conclusion lapidaire : "no statistically significant difference ${ }^{8}$. II importe également de tenir compte du fait que la perception d'une innovation - tant du côté des enseignants que des étudiants et de l'établissement - s'avère particulièrement subjective (ce qui est innovant pour l'un ne l'est pas forcément pour l'autre) et située (selon le contexte et surtout les pratiques individuelles courantes, la perception de l'innovation ne sera pas similaire).

\footnotetext{
${ }^{6}$ Pour une revue de synthèse approfondie du concept d'innovation dans une perspective pluridisciplinaire et de l'innovation pédagogique en particulier, y compris, pour ce qui concerne la seconde référence ci-après, dans l'enseignement supérieur, voir Cros (1997) et Béchard (2001).

${ }^{7}$ Voir, par exemple, l'ouvrage collectif dirigé par Charlier et Peraya (2002) intitulé Technologie et innovation en pédagogie. Dispositifs innovants de formation pour l'enseignement supérieur.

${ }^{8}$ C'est ce qui a d'ailleurs provoqué, dans les années 1990, le fameux débat entre Clark et Kozma qui a donné lieu à la formule lapidaire de Clark (1994, p. 22) : " media are mere vehicle that deliver instruction but do not influence student achievement any more than the truck that delivers our groceries causes changes in our nutrition ».
} 
Une autre difficulté apparaît lorsque l'on se penche sur la question plus spécifique de l'innovation technopédagogique. De nombreux travaux menés autour de l'introduction des technologies (notamment numériques) dans les activités d'enseignement et d'apprentissage abordent l'innovation sous un angle plus empirique que théorique, en rendant compte d'implémentations locales plutôt réussies qui valorisent le travail effectué et mettent en avant la satisfaction générale de ses acteurs et de ses bénéficiaires. Toutefois, ces études génèrent une illusion récurrente au sein de la sphère éducative au sens large : celle d'une filiation directe qui lierait de façon intrinsèque usage des technologies et innovation pédagogique. De nombreux acteurs - et notamment les décideurs institutionnels - ont en effet tendance à attribuer d'emblée à toute technologie nouvelle (ou « dernière-née », selon la formule de Jacquinot, 1977/2012, p. XV), implémentée dans l'un ou l'autre rouage du processus d'enseignement et d'apprentissage, une « valence d'innovation pédagogique » (Jacquinot, 1977/2012). L'exemple récent de l'engouement autour des cours en ligne ouverts et massifs (MOOC ou CLOM) est, à ce titre, assez emblématique (voir Daniel, 2012).

Certains chercheurs comme Fluckiger (2018) n'hésitent d'ailleurs pas à dénoncer cette forme de naïveté technodéterministe : « il n'y a aucune attestation empirique pas plus que de nécessité logique à ce que l'innovation technologique entraîne une innovation pédagogique ». Et de poursuivre en insistant : « II faut le redire avec force car le paradigme déterministe, bien que maintes fois démenti continue, envers et contre tous nos résultats de recherche, de constituer le cadre de pensée des décideurs, des marchands et des discours médiatiques » (Fluckiger, 2018, §4 et 5).

On le voit, la question de l'innovation pédagogique, sa définition, ce que l'on peut en attendre et l'évaluation de ses effets sont des questions complexes impliquant la considération de nombreux facteurs tels que l'expérience personnelle des acteurs, leurs représentations des technologies, leur degré d'aisance instrumentale, etc. La nécessité de distinguer la nature de l'innovation se pose de manière essentielle dès lors qu'il s'agit d'en évaluer les effets. Albero, Linard et Robin (2009) proposent, à cet égard, une analyse intéressante :

Si l'intégration des "TIC » est réduite à une affaire essentiellement technique, elle est assez rapidement résolue et ne change rien à la structure du système existant. Si elle est posée comme une question structurelle et organisationnelle, elle devient plus complexe, mais elle peut n'aboutir qu'à un réaménagement de l'existant. Si elle est posée comme une question politique et institutionnelle, elle peut être une chance pour l'université de répondre à des questions auxquelles d'autres organisations pourraient répondre sans elle si elle ne s'y intéresse pas (p. 138).

Le modèle socio-organisationnel proposé par Prost (2013) - et réinvesti par la suite par Peraya (2018) dans une perspective destinée à décrire l'innovation technopédagogique - reflète de façon plus « modélisante » l'analyse d'Albero, Linard et Robin (2009). Prost (2013) propose ainsi de distinguer trois niveaux de changements susceptibles d'atteindre la sphère éducative :

1) les changements mécaniques ne touchant ni à « l'architecture d'ensemble de l'organisation concernée ni à ses principes de gouvernement »;

2) les changements organiques, lesquels « affectent en revanche l'architecture, l'organisation d'ensemble, mais [...] n'altèrent pas les principes de fonctionnement, la logique selon laquelle le système se gouverne $»$; 
3) les changements structurels ou paradigmatiques qui « remettent en question les fondements, les principes mêmes d'organisation et de gouvernement de l'institution » (Prost, 2013, p. 303-304, cité par Peraya, 2018, §26).

La plupart des changements constatés à l'aune de l'introduction d'innovations technologiques et/ou pédagogiques sont le plus souvent de nature mécanique ou organique (Peraya, 2018). L'auteur va plus loin en considérant à ce titre que :

Le risque pour le processus d'innovation est sans aucun doute de voir certains de ces changements mécaniques se figer et devenir à leur tour une nouvelle norme, comme c'est le cas aujourd'hui pour les capsules vidéo produites pour les $M O O C$ qui, dans leur très grande majorité, ont sclérosé et renforcé dans une forme médiatique normalisée une conception transmissive, instructionniste de l'enseignement universitaire (Peraya, 2017; Peltier et Campion, 2017)

(Peraya, 2018, § 23).

La question de savoir à quelles conditions une innovation technopédagogique peut engendrer un changement de nature paradigmatique reste encore à examiner. L'une des pistes actuellement explorées (Peltier, Peraya, Bonfils et Heiser, à paraître) consiste, d'une part, à considérer la résistance " naturelle " des établissements d'enseignement au changement (Barouch, 2011) et, d'autre part, à envisager la mise à l'épreuve de l'innovation à travers un processus d'assimilation destiné à la rendre compatible avec les pratiques ancrées. En d'autres termes, la « menace » que peut faire peser l'introduction d'une nouveauté dans la routine des pratiques et l'inconfort que cette menace génère peut être réduite par une forme d'accommodation qui consiste à n'ajuster que le nécessaire pour ne pas perturber l'ensemble. Cette forme d' « institutionnalisation » des innovations est décrite par Céci $(2018, \S 6)$ comme " une légère adaptation structurelle au "désordre" créé par l'innovation pédagogique ». Les quelques exemples suivants proposés par Albero, Linard et Robin (2009, p. 148-149) illustrent bien ce point de vue: "les supports technologiques sont devenus d'usage courant pour les fonctions ordinaires d'administration (gestion des inscriptions, évaluations, certifications) et pour la diffusion électronique des informations et communications institutionnelles". Les formations dites hybrides (auxquelles on pourrait associer les $M O O C$ aujourd'hui ${ }^{9}$ ) jouent, selon les auteurs, " un rôle d'argument promotionnel ou de vitrine de modernité, sans pour autant entraîner de transformation profonde des modes traditionnels d'enseignement sur les campus ». Après cette première entrée en matière, venons-en à présent à ce qui constitue l'objet de ce texte : la diffusion de cours en direct.

\section{La télédiffusion de cours universitaires : une histoire déjà déjà longue}

La diffusion de cours en direct (ou « télédiffusion » selon la terminologie première) n'est pas une nouveauté dans le paysage académique. Dans son ouvrage consacré à l'histoire de la formation à distance, Glikman (2002) rappelle que ce que l'on désignait alors comme « la télévision éducative » est née dans les années

\footnotetext{
${ }^{9}$ Non pas pour leurs caractéristiques technopédagogiques, mais pour les conditions de leur développement, notamment du point de vue de leur promotion institutionnelle.
} 
1960 sous l'impulsion, en ce qui concerne la France, d'une volonté politique visant à " pallier les déficiences du système scolaire et, dans une moindre mesure, universitaire, en matière de locaux et d'enseignants » (Glikman, 2002, p. 170). Qualifiée d'« enseignement de la seconde chance », la formation à distance d'alors est principalement destinée à la formation d'adultes dans une perspective de lutte contre les inégalités sociales.

L'une des premières initiatives mondiales de diffusion de cours universitaires en direct est le fait du Conservatoire national des arts et métiers (CNAM) à Paris. Dès 1963, certains des cours magistraux dispensés au CNAM sont diffusés en direct, d'abord sur circuit fermé retransmis dans des centres régionaux, puis sur le réseau hertzien. Destinés à être suivis par un public dit "empêché ${ }^{10}$, ces cours sont d'abord retransmis dans leur forme simple (« cours filmé »), puis font l'objet de « mises en images plus élaborées » (« petit groupe d'apprenants qui dialoguent avec l'enseignant ») (Glikman, 2002, p. 176).

Les années 1970 verront la mise en place d'initiatives similaires ailleurs en France ${ }^{11}$, mais aussi en Grande-Bretagne, en Allemagne, au Canada et aux États-Unis ${ }^{12}$. Beaucoup de ces projets seront abandonnés, notamment pour des raisons de coûts de diffusion. L'avènement du Web dans les années 1990 change la donne et permet, entre autres, la mise en place d'une diffusion en flux continu (ou streaming ${ }^{13}$. Le début des années 2000 est marqué par la généralisation des plateformes pour l'enseignement et l'apprentissage à distance et la naissance d'un véritable " marché » de l'e-learning ${ }^{14}$. L'irruption de ces plateformes dans le paysage universitaire rendra possible la médiatisation d'un ensemble de fonctions génériques des dispositifs de formation: gestion, information, interaction, production, évaluation, métaréflexion, présence sociale (ou awareness) (Peraya, 2008). Dans la plupart des cas toutefois, seule la fonction d'information est exploitée par les enseignants ${ }^{15}$. C'est-à-dire que les étudiants ont accès à des éléments de contenu du cours (diaporamas, syllabus, lectures, etc.), mais ne sont pas engagés à utiliser la plateforme comme un espace de travail, de production et/ou d'interaction ${ }^{16}$. II s'agit là d'un premier exemple illustrant le fait qu'une innovation technologique peut, contrairement à ce qui est souvent mis en avant, renforcer, voire engendrer un retour à des pratiques d'enseignement exclusivement transmissives.

Si la captation et la mise à disposition des cours enregistrés sont un service offert aujourd'hui par de nombreuses universités (Peltier, 2016a), la diffusion des cours en direct était encore peu généralisée

\footnotetext{
10 Paquelin (2014) donne des étudiants « empêchés » la définition suivante : " dans l'incapacité de se rendre sur le campus pour suivre les cours magistraux et assister aux travaux dirigés en présentiel ».

11 D’après Glikman (2002), plusieurs universités (Strasbourg, Dijon, Nancy et Grenoble) s'appuieront ainsi sur le réseau de la troisième chaîne nationale (FR3) et sur ses relais régionaux pour proposer la télédiffusion de cours destinés à des étudiants « empêchés » de premier cycle.

12 Pour en savoir plus, voir Glikman (2002, p. 181-183).

${ }^{13}$ Voir Mortensen, Schlieve et Young (2000) pour les détails techniques de ces premières mises en place.

${ }^{14}$ Voir le répertoire des « plateformes de e-learning et de e-formations » tenu et mis à jour sur Thot Cursus depuis le début des années 2000 : https://cursus.edu/formations/17676\#.XYEDpH_gqM8

15 Plusieurs études, dont une menée à l'Université de Genève auprès de la Faculté des sciences (Peltier, 2010), font état de ces usages limités des plateformes d'enseignement et d'apprentissage. Un pointage ultérieur en 2013 effectué sur l'ensemble des facultés de l'Université de Genève a montré que la situation n'avait pas beaucoup évolué depuis (résultats non publiés).

${ }^{16}$ C'est ce que l'on a également pu observer durant les périodes de fermeture des universités en 2020 avec une mise à distance des cours universitaires centrée avant tout sur la médiatisation des contenus.
} 
jusqu'aux événements liés à la pandémie de COVID-19 ${ }^{17}$. Jusqu'alors, la diffusion en direct était généralement réservée aux "grands événements" (conférences de prestige, remise de diplômes, événements institutionnels). Certaines universités ${ }^{18}$ ont toutefois étendu cette pratique à la retransmission en direct de cours magistraux.

La diffusion de cours en direct se met souvent en place dans la continuité d'un service de captation et d'archivage de cours (Mullins-Dove, 2006, p. 69). C'est le cas, par exemple, de l'Université de Genève qui a développé ce dispositif dans le prolongement du premier et qui utilise la même infrastructure d'accès et d'archivage (Mediaserver) pour les deux types d'enregistrement (cours enregistrés et diffusés a posteriori et cours diffusés en direct et archivés). On pourrait dès lors être tenté de considérer ces deux services comme un ensemble et les analyser comme tel, tant sur le plan de leur intérêt pour l'apprentissage que sur celui des actions d'accompagnement à mettre en place pour favoriser leur appropriation. Or il convient de les différencier sur certains points. Tout d'abord parce que les conditions d'usage ne sont pas les mêmes ${ }^{19}$, mais surtout parce que le caractère synchrone des cours diffusés en direct constitue une occasion particulière d'amener les enseignants à se préoccuper de la façon d'intégrer les étudiants distants.

Tout comme la diffusion des cours en direct, l'enregistrement et l'archivage des cours universitaires sont un service qui, lui aussi, existait avant l'avènement des technologies numériques. Par exemple, l'Université de Genève enregistrait déjà, dès 1970, sur des cassettes audio analogiques, la plupart des cours donnés par sa Faculté des lettres (Burdet, Bontron et Burgi, 2007; Peltier, 2016a). II paraît intéressant de rappeler que cette initiative reposait sur une contrainte, mais aussi sur une nécessité. Tout d'abord, il s'agissait de permettre aux étudiants de suivre tous les cours de leur programme d'études, ce qui, pour certains d'entre eux en fonction de leurs choix spécifiques, s'avérait difficile en raison du chevauchement de certains horaires de cours. Les étudiants concernés pouvaient donc se rendre à la médiathèque de l'Université pour écouter a posteriori l'enregistrement sur place ou emprunter les cours manqués. Ce projet répondait également à une volonté institutionnelle de conserver une trace de ses cours les plus emblématiques.

17 Compte tenu des réactions négatives liées à des expériences d'enseignement et d'apprentissage insatisfaisantes (du fait, notamment, de la centration sur les contenus et d'un mode de communication avant tout transmissif et diffusionnel), il est peu probable que cette généralisation se maintienne.

${ }^{18}$ En Australie (par exemple Macquarie University), au Canada (par exemple University of Regina), au États-Unis (par exemple University of

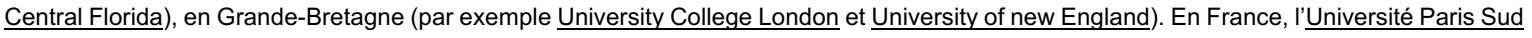
(Saclay) propose un tel service depuis plusieurs années, ainsi que l'Université Pierre et Marie Curie, l'Université Paris Est (Créteil) et l'Université d'Angers. Cette liste n'est pas exhaustive.

19 Pour l'une des deux modalités, l'écoute se fait en temps quasi réel, tandis que pour l'autre, l'écoute se fait en différé. Si la manipulation (appuyer sur pause, revenir en arrière) est possible dans les deux cas, la modalité live streaming permet toutefois moins de liberté à cet égard. 


\section{La diffusion des cours en direct : l'exemple de l'Université de Genève}

Le projet « Live streaming » a démarré en 2016 à l'Université de Genève ${ }^{20}$ avec la diffusion de trois cours ${ }^{21}$ de première année de baccalauréat universitaire. Chacun de ces cours concerne un domaine d'études différent : la médecine, le droit et l'économie. II s'agit de cours d'introduction générale, dont le suivi est obligatoire, et qui présentent la particularité de s'adresser à de très grands groupes d'étudiants (entre 500 et 700 selon les années). Si ces trois enseignements s'apparentent à des cours magistraux classiques (déroulement en auditoire sous la forme d'exposés réalisés par l'enseignant), l'approche pédagogique adoptée (notamment en matière d'activités d'apprentissage et de nature des interactions proposées aux étudiants) est toutefois différente d'un cours à l'autre, comme nous le verrons plus bas.

À la différence des cours enregistrés et mis à disposition a posteriori, les trois cours diffusés en direct proposent l'image de l'enseignant en petit médaillon au coin de l'écran. La littérature n'apporte pas de réponse univoque quant à la pertinence de conserver à l'écran l'image de l'enseignant lors d'un cours à distance. Homer, Plass et Blake (2008) ont par exemple établi que, dans les conditions expérimentales qu'ils ont mises en place, la présence ou l'absence de l'incrustation vidéo du visage de l'enseignant ne générait aucune différence significative sur l'apprentissage (mémorisation et compréhension ${ }^{22}$ ). Dans leur étude, les auteurs rendent également compte du fait que la présence conjointe de l'image, du son et du texte (diaporama) peut générer une surcharge cognitive ${ }^{23}$. Plusieurs études (par exemple Kizilcec, Papadopoulos et Sritanyaratana, 2014) évoquent toutefois la préférence des étudiants pour la modalité combinée image, son et visage de l'enseignant ("face in video instruction »). Cette préférence peut s'expliquer par la nécessité de la présence sociale, quelle que soit la situation d'enseignement et d'apprentissage, mais particulièrement en formation à distance (Peraya, Charlier et Deschryver, 2014).

Tout comme cela est le cas pour les cours enregistrés, la diffusion des cours en direct est automatique et ne requiert aucune intervention particulière de l'enseignant. Toutefois, selon le déroulement du cours et les activités envisagées, quelques aménagements peuvent s'avérer nécessaires. Par exemple, deux des enseignants impliqués dans la phase pilote du projet avaient pour habitude préalable l'utilisation de $P_{i n g o}{ }^{24}$, un dispositif interactif de vote en ligne. La présence des étudiants connectés à distance a demandé un ajustement dans la gestion des activités de vote afin de leur permettre de participer aux exercices proposés au même titre que les étudiants présents dans l'auditoire. On observe en effet un léger décalage (une vingtaine de secondes environ) entre ce qui est dit dans l'auditoire et ce qui est diffusé. Concrètement, il s'agit avant tout de prévoir un temps de latence suffisant entre le moment où la question est posée et le moment où les étudiants répondent, afin de permettre à l'ensemble d'entre eux de prendre connaissance de la question et de donner leur réponse. Les questions adressées aux étudiants peuvent

\footnotetext{
${ }^{20}$ Notons que l'Université de Genève est la première en Suisse à s'être dotée d'un tel dispositif.

${ }^{21}$ À partir de 2019, le dispositif a été étendu à tous les cours de médecine de première année, puis à d'autres cours de différentes facultés.

22 "Recall was tested using multiple-choice questions and transfer was tested using short-answer questions " (Homer, Plass et Blake, 2008, p. 790)

${ }^{23}$ II s'agit du phénomène de partage attentionnel (ou split attention effect) qui a été abondamment documenté, notamment par Moreno et Mayer à partir de la fin des années 1990 .

${ }^{24}$ https://trypingo.com/ (logiciel open source permettant le vote interactif et développé par l'Université de Paderborn, Allemagne).
} 
prendre la forme de questions à choix multiples ou de questions à réponses ouvertes avec l'affichage d'un nuage de mots dont la taille est relative à la fréquence des termes contenus dans les réponses données.

\section{Quelle forme d'innovation pédagogique?}

Une première évaluation du dispositif menée par Cacault, Hildebrand, Laurent-Lucchetti et Pellizzari (2019) a montré que la mise en place de ce service a permis de diminuer le nombre d'étudiants présents dans les auditoires d'environ $10 \%$, ce qui constituait, rappelons-le, l'objectif de la mise en place de la diffusion des cours en direct. L'étude a également montré que ce dispositif a avant tout profité aux étudiants tenus pour performants ${ }^{25}$ et $\mathrm{a}$, au contraire, défavorisé les étudiants considérés comme moins performants ${ }^{26}$. Conformément à ce qui avait déjà été observé quant aux usages des cours enregistrés (notamment par Peltier, Peraya, Grenon et Larose, 2016), l'étude a également permis de souligner que l'usage qu'en font les étudiants s'apparente plus à un usage supplétif (rattraper un cours manqué) qu'à un usage de substitution (ne plus se rendre en cours et le suivre uniquement selon cette modalité).

La diminution limitée du nombre d'étudiants présents dans les auditoires à la suite de l'implémentation de dispositifs tels que l'enregistrement des cours et/ou leur diffusion en direct peut s'expliquer par certains éléments que les études et les enquêtes n'abordent pas toujours. L'étude menée par Peltier, Peraya, Grenon et Larose (2016) a souligné une moindre propension des étudiants à faire usage des cours enregistrés lorsque les enseignements présentiels présentent une composante interactive élevée ${ }^{27}$. II semblerait donc que plus un enseignant favorise les interactions en face à face, voire les intègre au scénario de cours en prévoyant des activités d'apprentissage, notamment collaboratives, moins les risques de voir les auditoires se vider sont élevés lorsque les cours sont enregistrés. L'étude a également pointé le fait que même dans le cas d'un cours magistral ne comportant que des interactions minimales, les étudiants conservent leur attachement au cours présentiel pour des raisons que l'on pourrait qualifier de socio-affectives. En effet, se rendre en cours, ce n'est pas seulement suivre un enseignement, c'est aussi rencontrer ses pairs, s'approprier et exercer son « métier d'étudiant " (Coulon, 1997/2005), profiter des infrastructures (cafétéria, bibliothèques, espaces de travail), etc.

Si la question de l'impact que pourrait avoir la mise à disposition des cours enregistrés - et, par extension, la diffusion des cours en direct - sur l'assiduité des étudiants fait encore souvent débat, il semblerait toutefois que la plupart des résultats convergent vers le constat qu'un tel dispositif n'a pas d'effet particulier sur la présence ou l'absence des étudiants. Les éventuelles différences observées pourraient toutefois

\footnotetext{
${ }^{25}$ L'indicateur utilisé pour qualifier la performance des étudiants (high ability students et low ability students) a été établi sur la base des résultats obtenus à la fin de l'école secondaire supérieure et donc à l'entrée à l'université.

${ }^{26}$ La littérature (notamment, Jézégou, 2008; Cosnefroy, 2012) a montré de longue date l'importance de l'autonomie et de l'auto-régulation dans les apprentissages. Celles-ci sont d'autant plus nécessaires en situation de formation à distance. Ainsi, les étudiants qui sont déjà dotés de telles compétences ne présentent pas les mêmes difficultés que les étudiants qui ne les ont pas encore acquises. Or la question de l'autonomie dans les apprentissages est souvent considérée comme un « allant de soi » qui ne nécessite pas d'être accompagnée.

${ }^{27}$ L'étude menée par Peltier, Peraya, Grenon et Larose (2016) a, par exemple, montré que : « Les étudiants rapportent l'importance que revêtent pour eux les activités présentielles : utilisation de boîtiers de vote interactifs, travaux collaboratifs, utilisation de la visioconférence, etc. Le cours 74111 scénarise les séances présentielles, autant que les phases à distance. Dans cette perspective, l'enregistrement des séances de cours lui fait perdre une grande partie de son intérêt; l'essentiel se déroule en présence, notamment à travers les activités collectives, les interactions, les productions collaboratives en ligne, etc. » (p. 69).
} 
être mises sur le compte des caractéristiques propres aux cours, ce qui, malheureusement, est rarement étudié dans les recherches consacrées à ces questions ${ }^{28}$.

Selon les enseignants concernés par la phase pilote du projet ${ }^{29}$, la diffusion des cours en direct n'a pas eu d'impact particulier sur le travail de préparation et le déroulement des cours. Ce constat général d'un " changement dans la continuité », qui pourrait paraître décevant au regard de la promesse de renouvellement des pratiques pédagogiques traditionnellement mise en avant lors de l'implémentation d'initiatives de ce genre, constitue au contraire, aux yeux de ses promoteurs, un argument pour rallier l'ensemble du corps enseignant à l'adoption du dispositif. On peut en revanche se questionner sur l'impact (positif ou négatif) qu'un tel dispositif pourrait avoir sur les étudiants.

Nous avons souligné plus haut que pour être considérée comme une innovation pédagogique, une nouveauté devrait procurer une amélioration substantielle à l'apprentissage (pour autant que l'on en définisse les contours et la façon d'en appréhender les effets). Dans cette perspective, on peut se questionner sur ce qui, dans la diffusion des cours en direct, relève d'une innovation (techno)pédagogique. Indéniablement, et les résultats empiriques ${ }^{30}$ et expérimentaux ${ }^{31}$ en attestent, l'implémentation de ce dispositif à l'Université de Genève a contribué à résoudre (partiellement et provisoirement du moins) le problème de la surcharge des auditoires. On peut donc considérer que d'un point de vue logistique et organisationnel, le dispositif a tenu ses promesses. On peut également considérer que le confort d'étude fait partie intrinsèque du processus d'apprentissage et qu'à ce titre l'impact positif de la diffusion des cours en direct sur la fréquentation des auditoires représente une amélioration des conditions d'apprentissage ${ }^{32}$.

La diversification des modalités d'accès à l'information et la flexibilité en matière de suivi des cours constitue également un plus pour les étudiants.

II convient toutefois de lever toute ambiguïté sur le fait que la diffusion des cours en direct ne constitue qu'une forme rudimentaire de cours à distance et ne peut être véritablement considéré comme tel, que la réécoute d'un cours a posteriori, si elle peut présenter un certain nombre d'avantages (combler une incompréhension, rafraîchir un souvenir imprécis, rattraper un cours manqué, etc.), comporte également un certain nombre de risques (par exemple la procrastination ${ }^{33}$ ) et de désagréments (comme un temps d'écoute plus long dû à la possibilité de manipuler l'enregistrement).

\footnotetext{
${ }^{28}$ II s'agit là de l'une des difficultés soulignées précédemment : les recherches portant sur les effets des technologies ne tiennent souvent pas compte du contexte et de ses spécificités, notamment des spécificités pédagogiques (approche, activités, orientation, etc.) des cours observés.

${ }^{29}$ Communications personnelles (juin 2019).

${ }^{30}$ Ceux des observations menées par la Faculté de médecine.

${ }^{31}$ Ceux de l'étude publiée par Cacault, Hildebrand, Laurent-Lucchetti et Pelizzari (2019).

32 Dans leur étude déjà citée à plusieurs reprises, Cacault, Hildebrand, Laurent-Lucchetti et Pelizzari (2019) évoquent d'ailleurs des " pics » d'utilisation du live streaming lorsque la météo est défavorable (" students are almost twice as likely to log into the streaming platform on bad days than on regular days» (p. 20)) et n'encourage sans doute pas le déplacement extérieur. Une mesure similaire a été appliquée aux périodes d'épidémie de grippe et a montré également un lien significatif.

${ }^{33}$ La littérature (notamment Nadeau, Senécal et Guay, 2003, p. 97) a montré que « la procrastination académique est un comportement répandu et nuisible au cheminement et au succès académique des étudiants de tous les niveaux ». Elle est également considérée comme un indicateur de difficultés en matière d'autorégulation des apprentissages (Jézégou, 2019).
} 
Pour en revenir à la qualification de l'innovation que pourrait constituer la diffusion des cours en direct, on pourrait considérer, en s'appuyant sur le modèle de Prost évoqué plus haut et en prenant appui sur l'exemple présenté, que l'on se situe vraisemblablement ici dans une forme de changement de type mécanique (aucune modification autre que celle de rendre disponible un enregistrement afin de permettre aux étudiants de suivre le cours à distance), voire organique (aménagement du déroulement du cours en tenant compte du léger décalage temporel de la diffusion lors des activités de vote interactif). Un changement de type paradigmatique entraînerait, comme son nom l'indique, une modification du modèle de référence, et donc un changement dans la manière de concevoir et de pratiquer l'enseignement. Dans ce contexte précis, cela impliquerait sans doute une autre façon d'envisager la coprésence d'étudiants en auditoire et à distance (notamment en matière d'interactions, d'activités d'apprentissage, d'accompagnement, de scénarisation, etc.). L'émergence récente des cours comodaux (ou HyFlex courses en anglais) constitue sans nul doute une perspective à suivre dans la mesure où, dans sa conception initiale (Beatty, 2006 et 2007), un cours comodal doit offrir aux étudiants des activités d'apprentissage équivalentes, quelle que soit la modalité de participation choisie, aucune ne constituant un étalon par défaut : présentiel, distance synchrone, distance asynchrone. L'organisation d'activités d'apprentissage (discussions, études de cas, évaluations formatives, etc.) constitue donc une caractéristique constitutive d'un cours comodal.

Comme nous l'avons souligné à travers notre revue de la littérature consacrée à l'innovation pédagogique, la diffusion des cours en direct peut donc venir s'inscrire dans la continuité de pratiques d'enseignement éprouvées et appréciées comme telles par un enseignant - et ses étudiants - sans les modifier pour autant. Cela ne constitue évidemment pas un point négatif, mais illustre, si besoin était, que l'introduction d'un élément nouveau dans un processus déjà bien ancré peut aisément être accommodée sans perturber l'ensemble. En revanche, accompagner la mise en place d'un tel service par une incitation à la réflexion autour des pratiques d'enseignement et au partage formel et informel de pratiques avec d'autres constitue une occasion intéressante de faire évoluer les pratiques d'enseignement à l'université. II convient également de ne pas considérer l'autonomie des étudiants face à de nouvelles modalités d'apprentissage comme un allant de soi, mais comme un impératif à accompagner.

\section{Conclusion}

Depuis le début de la crise sanitaire mondiale de COVID-19 en mars 2020, la formation à distance et l'usage pédagogique des technologies numériques sont au centre de nombreuses préoccupations. Toutefois, la propension à considérer la formation à distance comme le pis-aller de la formation présentielle classique $^{34}$, assortie d'une vision "mécaniste » des technologies, contribue malheureusement à la cristallisation de représentations erronées qui risquent de desservir à long terme la formation à distance. Souligner sans relâche que la mise à distance totale ou partielle d'un dispositif de formation nécessite davantage que la médiatisation des contenus d'apprentissage (et, dans le meilleur des cas, des évaluations), c'est rappeler qu'au cours de son histoire, la formation à distance a connu différents développements et qu'aujourd'hui c'est l'ensemble des fonctions propres à tout dispositif de formation

\footnotetext{
${ }^{34}$ Dans une interview donnée à France Info le 26.11.2020, le président de l'Université de Strasbourg qualifiait le " télé-enseignement » de " bonne roue de secours » qui ne « remplacera jamais le présentiel ». (https://tinyurl.com/4m3hynku)
} 
(Peraya, 2008) qu'il convient de médiatiser afin d'offrir aux apprenants une expérience d'apprentissage riche et engageante fondée sur un paradigme centré sur l'activité et les interactions. À l'heure où l'on évoque abondamment, et à juste titre, le lien pédagogique et le lien social comme étant des conditions sine qua non de l'apprentissage, les dispositifs de formation à distance mis en place sont pourtant invariablement centrés sur les contenus dans une perspective transmissive et diffusionnelle des savoirs. II y a là une contradiction qui n'est pas le fait de la formation à distance et des technologies numériques, mais de connaissances limitées en la matière et fondées sur le sens commun. La réhabilitation de l'histoire du domaine comme une source indispensable aux réflexions d'aujourd'hui et de demain permettrait d'aborder les innovations de manière beaucoup plus fine et, surtout, éclairée.

\section{Liste de références}

Albero, B. (2014). La pédagogie à l'université entre numérisation et massification. Apports et risques d'une mutation. Dans G. Lameul et C. Loisy (dir.), La pédagogie universitaire à l'heure du numérique (p. 25-53). Bruxelles : De Boeck.

Albero, B., Linard, M. et Robin, J.-Y. (2009). Petite fabrique de l'innovation à l'université. Quatre parcours de pionniers. Paris : L'Harmattan.

Beatty, B. J. (2006). Designing the HyFlex World- Hybrid, Flexible Classes for All Students. Paper presented at the 2006 Association for Educational Communication and Technology International Conference, Dallas, TX.

Beatty, B. J. (2007, octobre). Hybrid classes with flexible participation options. If you build it. How will they come? Communication présentée à la Convention annuelle de l'Association for Educational Communications and Technology, Anaheim CA.

Béchard (2001). L'enseignement supérieur et les innovations pédagogiques : une recension des écrits. Revue des sciences de l'éducation, 27(2), p. 257-281.

Bédard, D. et Béchard, J.-P. (dir.) (2009). Innover dans l'enseignement supérieur. Paris : Presses universitaires de France.

Burdet, B., Bontron, C. et Burgi, P.-Y. (2007). Lecture Capture: What Can Be Automated? Educause Quarterly, 30(2), p. $40-48$.

Cacault, P., Hildebrand, C., Laurent-Lucchetti, J. et Pellizzari, M. (2019). Distance Learning in Higher Education: Evidence from a Randomized Experiment. Discussion Papers Series IZA Institute of Labor Economics, IZA DP nº 12298.

Céci, J.-F. (2018). Les technologies peuvent-elles modifier la forme universitaire ? Certainement ! Distances et médiations des savoirs, 22. https://journals.openedition.org/dms/2360

Chaptal, A. (1996). Technologies éducatives : des invariants à méditer. Communication présentée aux $1^{\text {res }}$ Rencontres de l'ORME, Marseille, France.

Charle, C. et Verger, J. (2012). Histoire des universités. XII $-X X I^{e}$ siècle. Paris : Presses universitaires de France.

Charlier, B. et Peraya, D. (2002). Technologie et innovation en pédagogie : dispositifs innovants de formation pour l'enseignement supérieur. Bruxelles : De Boeck Université.

Clark, R. E. (1994). Media Will Never Influence Learning. Educational Technology Research and Development, 42(2), p. 21-29.

Coulon, A. (1997/2005). Le métier d'étudiant : l'entrée dans la vie universitaire. Paris : Presses universitaires de France.

Cosnefroy, L. (2012). Autonomie et formation à distance. Recherche \& Formation, 69, p. 111.118.

Cros, F. (1997). L'innovation en éducation et en formation. Revue française de pédagogie, 118, p. 127-156.

Daniel, J. (2012). Making Sense of MOOCs: Musings in a Maze of Myth, Paradox and Possibility. Journal of Interactive Media in Education, 18.

Endrizzi, L. et Sibut, F. (2015). Les nouveaux étudiants d'hier à aujourd'hui. Lyon : Institut français de l'éducation.

Fluckiger, C. (2018). La forme universitaire comme analyseur des « effets » de la technologie : perspective critique. Distances et médiations des savoirs, 22. https://doi.org/10.4000/dms.2329

Glikman, V. (2002). Des cours par correspondance au « e-learning ». Paris : Presses universitaires de France. 